\title{
Floral diversity of District Bagh, Azad Jammu and Kashmir Pakistan
}

\author{
Muhammad Tanvir ${ }^{1}$, Ghulam Murtaza ${ }^{1}$, Khawaja Shafique Ahmad ${ }^{2, *}$, Muhammad Salman $^{3}$ \\ 1Department of Botany, University of Azad Jammu \& Kashmir, Muzaffarabad \\ ${ }^{2}$ Department of Botany, University of Agriculture, Faisalabad, Pakistan \\ ${ }^{3}$ Department of Plant Breeding and Genetics, University of Agriculture, Faisalabad, Pakistan \\ *Corresponding author:khsahmad@yahoo.com
}

Copyright (C) 2014 Horizon Research Publishing All rights reserved.

\begin{abstract}
The study showed the floral diversity and variation in the structure of vegetation in District Bagh, AJ\&K. A total of 200 plants belong to 65 families and 170 genera were documented from seven localities. The most species richest site was found Mahmood Galli with 101 species (dicots 55, monocots 35, pteridophytes 10 and gymnosperm 1) followed by Toliper $100 \mathrm{spp}$. (dicots 55, monocots 31 , pteridophytes 13 and gymnosperms 1), Lasdana, 85 spp. (dicots 47 , monocots 35 , pteridophytes 6 and gymnosperms 2), Plungi 81 spp. (dicots 51, monocots 13 , pteridophytes 16 and gymnosperm 1), Khurshidabad, 74 spp. (dicots 34, monocots 21, pteridophytes 19), Kahutta, 70 spp. (dicots 36, monocots 15, pteridophytes 19 ) and Kiran 46 Spp. dicots 34, gymnosperm 3). Among pteridophytes, Polystichum squarrosum had highest distribution percentage $85.71 \%$ while Lepisorus clathratus and Pyrrosia mollis have least percentage distribution $4.28 \%$. Among gymnosperms, Pinus wallichiana had highest distribution percentage $57.14 \%$ while Cedrus deodara and Pinus roxburgii have least distribution percentage 14.28. Aristida abnormis had highest distribution percentage with $71.42 \%$ while Zizania aquatica and Themeda anathera have least distribution percentage with $14.28 \%$. In dicots, Amaranthus viridis, Amaranthus spinosus and Cucumis sativus had highest distribution percentage with $85.71 \%$ and Oxalis corniculata, Quercus incana and Fragaria nubicola had least frequency with $14.28 \%$. Species diversity was high in the tree layer in the middle part of the altitudinal gradient. It decreases both towards upper and lower altitude, which was due to deforestation, human interaction, encroachment pressure, low number of species and soil erosion.
\end{abstract}

Keywords Floral Diversity, Distribution, Distric Bagh, AJ\&K

\section{Introduction}

Composition, distribution and classification of plant communities are the spirit of vegetation science for centuries, [1]. Altitude, aspect and annoyance from human settlements have linear relationship with vegetation attributes such as species richness, diversity and maturity [2]. Topography is the principal controlling factor in vegetation growth and that the type of soils and the amount of rainfalls play secondary roles at the scale of hill slopes. Elevation, aspect, and slope are the three main topographic factors that control the distribution and patterns of vegetation in mountain areas [3]. Several studies acknowledge the relationship among plant species diversity, richness, climate and spatial variables in the area $[4,5]$. The identification and description of local flora is very important, because it can show specific species of the local area and their occurrence, growing season, species hardness, distinct species, finding new species and the effect of climatic conditions like drought and over-grazing on vegetation [6].

An effective conservation plan cannot be implemented without knowing the status of indigenous plant species, ecology of habitat types, and factors affecting the population of plant species, particularly those of vulnerable and threatened either locally or internationally [7]. Knowledge on biodiversity of the study area is still fragmentary and requires deep studies to disclose all of its components. Flora of the Bagh region is currently under heavy pressure like anthropogenic activities, population pressure and grazing pressure. The objective of this study was to reveal the concealed anthropogenic and environmental factors disturbing the plant species diversity and richness. Present study will be helpful to ecologists, conservationists, forest managers and future researchers to compare any change in the species and species composition of plant communities of this hilly area.

\section{Materials and Methods}

\subsection{The Study Area}

The state of Azad Jammu and Kashmir is located between 
$73^{\circ}-75^{\circ}$ longitude and $33^{\circ}-36^{\circ}$ latitude (figure 1). It is located in the Pirpanjal sub range of the western Himalayan foothills. The total area of District Bagh is 1368 square kilometres which is about $10 \%$ of total land area of Azad Jammu \& Kashmir. Average annual temperature is $21^{\circ} \mathrm{C}$, ranging from $2^{\circ} \mathrm{C}$ in January to $40^{\circ} \mathrm{C}$ in July. The annual precipitation is about $1500 \mathrm{~mm}$ [8].

\subsection{Field Surveys}

Extensive vegetation surveys of different ecological zones ranging from subtropical to alpine zones were conducted during 2009-10 to assess the floral diversity and community structure in the District Bagh. The area under research had great altitudinal variation exhibiting different zones such as subtropical, temperate and alpine. Seven different localities including Tauliper, Lasdana, Mahmood Gali, Khurshidabad, Plungi, Kahutta and Kiran were selected, considering altitudinal variations.

\subsection{Vegetation Sampling}

Quadrat method was used for vegetation sampling. A transect of $200 \mathrm{~m}$ length was used at each sampling site, where each sampling point was separated by $50 \mathrm{~m}$ from the next. Ten quadrats $\left(10 \mathrm{~m}^{2}\right.$ for trees, $5 \mathrm{~m}^{2}$ for shrubs and $1 \mathrm{~m}^{2}$ for herbs and grasses) were laid perpendicularly along a straight line at each sampling point, 5 on each side of the sampling point. Plant specimens were critically examined and identified with the help of literature and authentic specimens at the Department of Botany University of Azad Jammu and Kashmir. Flora of Pakistan [9, 10], was followed for the proper identification. The number of the plants scored with reference to its ecological amplitude, occurrence, distribution and relative frequency of plant species were listed in (Table 1). The collected specimens were deposited in herbarium Department of Botany University of Azad Jammu and Kashmir. The percentage distribution of plant species in different localities was determined by using following formula;

$$
\text { Percentage Distribution }=\frac{\text { No of Localities in which a Plant Occurs }}{\text { Total No .of Localities }} \times 100
$$

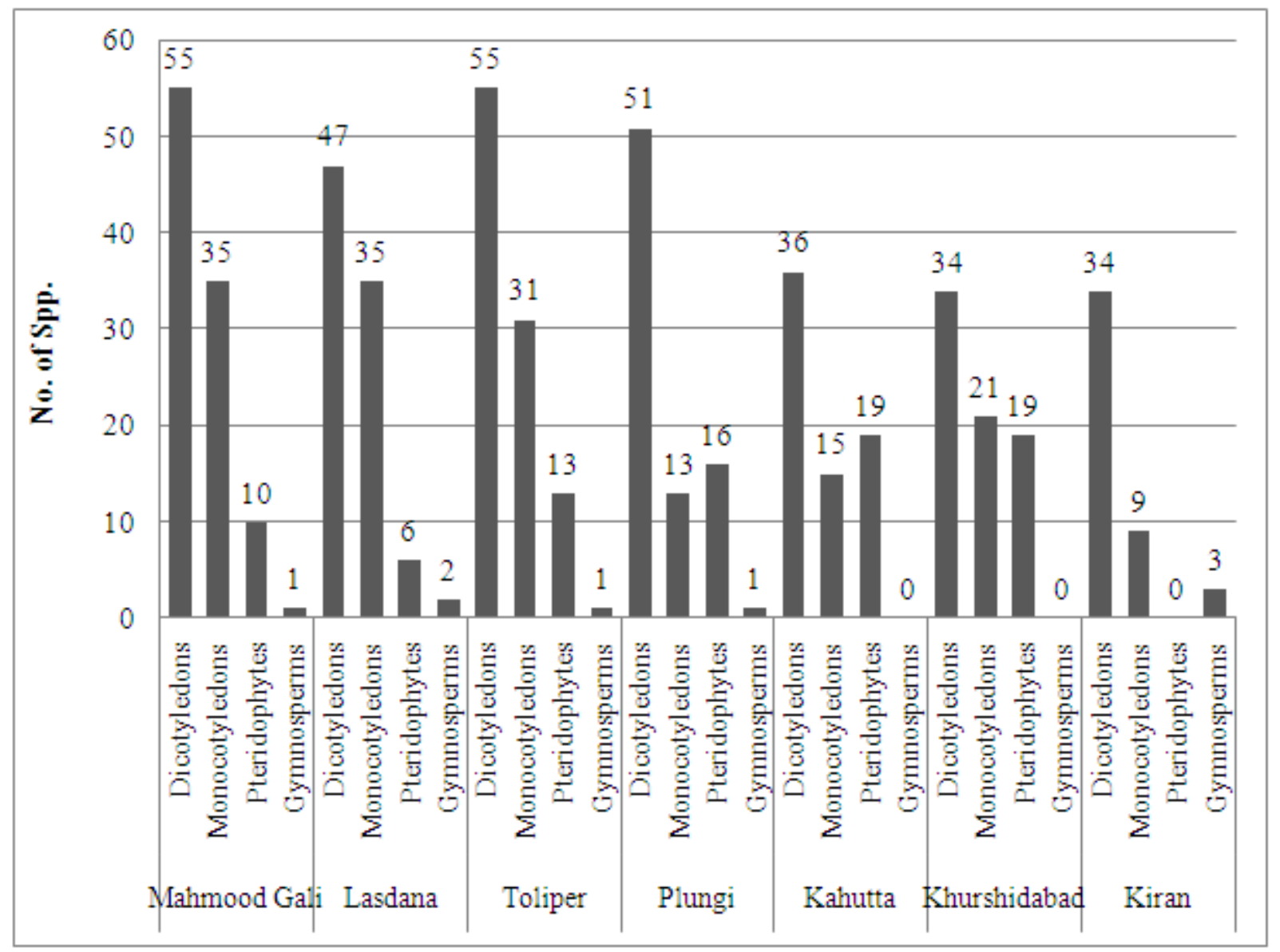

Figure 1. Plant groups distribution at 7 localities in the District Bagh, AJ\&K 
Table 1. Occurrence and Percentage distribution of plants in Distric Bagh, AJ\&K

\begin{tabular}{|c|c|c|c|c|c|c|c|c|c|c|c|c|}
\hline \multirow[t]{2}{*}{$\mathrm{S} \#$} & \multirow{2}{*}{$\begin{array}{l}\text { Plant groups } \\
\text { Pteridophytes }\end{array}$} & \multirow[t]{2}{*}{ Family } & \multirow[t]{2}{*}{ Habit } & \multicolumn{7}{|c|}{ Distribution } & \multirow[t]{2}{*}{ Distribution (\%) } & \multirow[t]{2}{*}{ Relative frequency (\%) } \\
\hline & & & & $\mathrm{L}_{1}$ & $\mathrm{~L}_{2}$ & $\mathrm{~L}_{3}$ & $\mathrm{~L}_{4}$ & $\mathrm{~L}_{5}$ & $\mathrm{~L}_{6}$ & $\mathrm{~L}_{7}$ & & \\
\hline 1 & Lycopodium selago L. & Lycopodiaceae & Herb & - & + & + & - & - & + & - & 42.85 & 0.54 \\
\hline 2 & Selaginella chrysocaulos (Hook. \& Grev.) Spring & Selaginellaceae & Herb & + & + & - & - & + & - & - & 42.85 & 0.54 \\
\hline 3 & S. sanguinolenta (L.) Spring & & Herb & - & - & - & + & - & + & - & 28.57 & 0.36 \\
\hline 4 & Equisetum arvense $\mathrm{L}$ & Equisetaceae & Herb & - & - & - & + & + & + & - & 42.85 & 0.54 \\
\hline 5 & E. diffusum D.Don & & Herb & - & - & - & + & + & + & - & 42.85 & 0.54 \\
\hline 6 & Ophioglossum retculatum $\mathrm{L}$. & Ophioglossaceae & Herb & - & - & - & + & + & + & - & 42.85 & 0.54 \\
\hline 7 & O. vulgatum $\mathrm{L}$. & & Herb & - & + & + & + & + & - & - & 57.14 & 0.72 \\
\hline 8 & Lygodium japonicum (Thunb.) Sw. & Schizaceacae & Herb & + & + & + & - & - & - & - & 42.85 & 0.54 \\
\hline 9 & Adiantum capillus- veneris $\mathrm{L}$. & Adiantaceae & Herb & - & - & - & - & + & + & - & 28.57 & 0.36 \\
\hline 10 & A. venustum D.Don & & Herb & - & - & - & + & + & - & - & 28.57 & 0.36 \\
\hline 11 & Coniogramme affinis Wal (C.presl) Hieron & & Herb & - & - & - & + & + & - & - & 28.57 & 0.36 \\
\hline 12 & C. caudata (Wall.) Ching & & Herb & - & - & - & + & + & - & - & 28.57 & 0.36 \\
\hline 13 & Onychium japonicum (Thunb.) Kunze & & Herb & - & - & + & + & + & - & - & 42.85 & 0.54 \\
\hline 14 & Pteridium aquilinum (L.) Kuhn & Polypodiaceae & Herb & + & + & - & - & + & + & - & 57.14 & 0.72 \\
\hline 15 & P. vittata $\mathrm{L}$. & Pteridaceae & Herb & - & + & + & + & - & - & - & 42.85 & 0.54 \\
\hline 16 & Athyrium acrostichoides $\mathrm{Sw}$. & & Herb & + & + & - & - & - & - & - & 28.57 & 0.36 \\
\hline 17 & A. dentigerum (Wall. ex C.B. Clarke) Mehra & & Herb & - & + & + & + & + & + & - & 71.42 & 0.90 \\
\hline 18 & A. schimperi Moug. Ex Fee & & Herb & - & - & + & + & + & + & - & 57.14 & 0.72 \\
\hline 19 & Dryopteris barbigera (T.Moore ex Hook.) Kuntze & Dryopteridaceae & Herb & - & - & + & + & + & - & - & 42.85 & 0.54 \\
\hline 20 & Hypodematium crenatum (Forssk.) Kuhn & & Herb & - & - & - & + & - & + & - & 28.57 & 0.36 \\
\hline 21 & Polystichum discretum (D.Don) J.Sm. & & Herb & + & + & - & - & - & + & - & 42.85 & 0.54 \\
\hline 22 & P. squarrosum (D.Don) Fee & & Herb & + & + & + & + & + & + & - & 85.71 & 1.08 \\
\hline 23 & Asplenium pseudofontanum Kossinsky & Aspleniaceae & Herb & - & - & + & + & + & + & - & 57.14 & 0.72 \\
\hline 24 & A. Trichomanes $\mathrm{L}$. & & Herb & - & - & - & + & + & - & - & 28.57 & 0.36 \\
\hline
\end{tabular}




\begin{tabular}{|c|c|c|c|c|c|c|c|c|c|c|c|c|}
\hline 25 & A. dalhousiae Hook. & & Herb & - & - & + & - & - & + & - & 28.57 & 0.36 \\
\hline 26 & Lepisorus clathratus (C.B. Clarke) Ching & Polypodiaceae & Herb & - & - & + & - & - & - & - & 14.28 & 0.18 \\
\hline 27 & L. nudus (Hook.) Ching & & Herb & - & - & - & + & + & + & - & 42.85 & 0.54 \\
\hline 28 & Pyrrosia mollis (Kunze) Ching & & Herb & - & - & + & - & - & - & - & 14.28 & 0.18 \\
\hline 29 & Marsilea minuta $\mathrm{L}$. & Marsiliaceae & Herb & - & - & - & + & + & + & - & 42.85 & 0.54 \\
\hline \multicolumn{13}{|c|}{ Gymnosperms } \\
\hline 30 & Pinus roxburgii Sarg. & Pinaceae & Tree & + & - & - & - & - & - & + & 14.28 & 0.18 \\
\hline 31 & Abies pindrow (Royle ex D.Don) Royle & & Tree & - & - & - & - & - & - & + & 28.57 & 0.36 \\
\hline 32 & Cedrus deodara (Roxb. ex Lamb.) G. Don & & Tree & - & - & - & - & - & - & + & 14.28 & 0.18 \\
\hline 33 & Pinus wallichiana A. B. Jacks. & & Tree & + & + & + & - & - & + & - & 57.14 & 0.72 \\
\hline \multicolumn{13}{|c|}{ Monocotyledon } \\
\hline 34 & Arisaema jacquemontii Blume & Araceae & Herb & & & & & & & & 57.14 & 0.72 \\
\hline 35 & Iris germanica $\mathrm{L}$. & Iridaceae & Herb & + & + & + & - & - & - & + & 28.57 & 0.36 \\
\hline 36 & Aristida funiculata Trin. & Poaceae & Herb & + & + & - & - & - & - & - & 57.14 & 0.72 \\
\hline 37 & A. abnormis Chiov. & & Herb & + & + & + & - & - & - & + & 71.42 & 0.90 \\
\hline 38 & Brachiaria ramose (L.) Stapf. & & Herb & + & + & + & + & + & - & - & 71.42 & 0.90 \\
\hline 39 & B. deflexa Schumach & & Herb & + & + & + & + & + & - & - & 28.57 & 0.36 \\
\hline 40 & Calamagrostis decora Hook. & & Herb & + & + & - & - & - & - & - & 71.42 & 0.90 \\
\hline 41 & Danthonia cachemyriana (Jaub) Spach & & Herb & + & + & + & + & + & - & - & 71.42 & 0.90 \\
\hline 42 & D. schneideri Pilger & & Herb & + & + & + & + & + & - & - & 28.57 & 0.36 \\
\hline 43 & Glyceria tonglensis (Clarke) L. & & Herb & + & + & - & - & - & - & - & 28.57 & 0.36 \\
\hline 44 & G. plicata (Fries) Fries. & & Herb & + & + & - & - & - & - & - & 71.42 & 0.90 \\
\hline 45 & Melica persica Kunth & & Herb & + & + & + & + & + & - & - & 57.14 & 0.72 \\
\hline 46 & Microstegium nudum Trin & & Herb & + & + & + & - & - & - & + & 71.42 & 0.90 \\
\hline 47 & Phacelurus speciosus Steud & & Herb & + & + & + & + & + & - & - & 28.57 & 0.36 \\
\hline 48 & Phleum alpinum L. & & Herb & + & + & - & - & - & - & - & 71.42 & 0.90 \\
\hline 49 & Piptatherum hilariae Pazij. & & Herb & + & + & + & + & + & - & - & 28.57 & 0.36 \\
\hline
\end{tabular}




\begin{tabular}{|c|c|}
\hline 50 & P. gracile Mez. \\
\hline 51 & Stipa capillata $\mathrm{L}$. \\
\hline 52 & S. breviflora Griseb. \\
\hline 53 & Steria pumila (Poir) Roem \& Schult \\
\hline 54 & S. viridis \\
\hline 55 & Cynodon dactylon (L.) Pers. \\
\hline 56 & Dactylis glomerata $\mathrm{L}$. \\
\hline 57 & Chrysopogon serrulatus Trin. \\
\hline 58 & C. aucheri (Boiss.) Stapf \\
\hline 59 & Digitaria sanguinalis (L.) Scop. \\
\hline 60 & Роа аппиа $\mathrm{L}$. \\
\hline 61 & P. neplensis Wall. ex Duthie. \\
\hline 62 & Zizania aquatica $\mathrm{L}$. \\
\hline 63 & Dichanthium annulatum (Forssk.) Staf \\
\hline 64 & Phalaris minor Retz. \\
\hline 65 & Themeda anathera (Nees ex Steud.)Hack. \\
\hline 66 & Avena sativa $\mathrm{L}$. \\
\hline 67 & Phragmites australis (Cav.) Trin. ex Steud. \\
\hline 68 & Andropogon gerardii Vitman \\
\hline 69 & Heteropogon contortus (L.) P. Beu. ex R. \& Sch \\
\hline 70 & Stipa orientalis Trin. \\
\hline 71 & Panicum miliaceum $\mathrm{L}$. \\
\hline 72 & Agrostis viridis Gouan \\
\hline 73 & A. hissarica Rozhev. \\
\hline 74 & Alopecurus himalaicus Hook \\
\hline 75 & A. aequalis Sobol. \\
\hline 76 & Arundo donax L. \\
\hline
\end{tabular}

\begin{tabular}{|c|c|c|c|c|c|c|c|c|c|}
\hline Herb & + & + & - & - & - & - & - & 71.42 & 0.90 \\
\hline Herb & + & + & + & + & + & - & - & 71.42 & 0.90 \\
\hline Herb & + & + & + & + & + & - & - & 71.42 & 0.90 \\
\hline Herb & + & + & + & + & + & - & - & 28.57 & 0.36 \\
\hline Herb & + & + & - & - & - & - & - & 28.57 & 0.36 \\
\hline Herb & + & + & - & - & - & - & - & 42.85 & 0.54 \\
\hline Herb & - & - & + & + & - & + & - & 28.57 & 0.36 \\
\hline Herb & - & - & + & - & - & + & - & 28.57 & 0.36 \\
\hline Herb & + & + & - & - & - & - & - & 71.42 & 0.90 \\
\hline Herb & + & + & + & + & + & - & - & 57.14 & 0.72 \\
\hline Herb & + & + & - & - & + & + & - & 57.14 & 0.72 \\
\hline Herb & + & + & + & - & - & + & - & 71.42 & 0.90 \\
\hline Herb & + & + & + & + & + & - & - & 14.28 & 0.18 \\
\hline Herb & - & - & - & - & - & - & + & 57.14 & 0.72 \\
\hline Herb & + & + & + & - & - & + & - & 57.14 & 0.72 \\
\hline Herb & + & + & + & + & - & + & - & 14.28 & 0.18 \\
\hline Herb & + & - & - & - & - & - & + & 14.28 & 0.18 \\
\hline Herb & - & - & - & - & - & - & + & 57.14 & 0.72 \\
\hline Herb & + & + & + & - & - & + & - & 14.28 & 0.18 \\
\hline Herb & - & - & - & - & - & - & + & 42.85 & 0.54 \\
\hline Herb & - & - & + & + & - & + & - & 28.57 & 0.36 \\
\hline Herb & + & + & - & - & - & - & - & 42.85 & 0.54 \\
\hline Herb & + & + & + & - & - & - & - & 28.57 & 0.36 \\
\hline Herb & + & + & - & - & - & - & - & 42.85 & 0.54 \\
\hline Herb & - & - & + & + & - & + & - & 28.57 & 0.36 \\
\hline Herb & - & + & + & - & - & - & - & 42.85 & 0.54 \\
\hline Herb & - & - & + & + & - & + & - & 42.85 & 0.54 \\
\hline
\end{tabular}




\begin{tabular}{|c|c|c|c|c|c|c|c|c|c|c|c|c|}
\hline 77 & Briza media $\mathrm{L}$. & & Herb & - & - & + & + & - & + & - & 14.28 & 0.18 \\
\hline 78 & Bromus inermis Leyss. & & Herb & - & - & - & - & - & - & + & 42.85 & 0.54 \\
\hline 79 & Carex brunnea Thunb. & Cyperaceae & Herb & + & + & + & - & - & - & - & 14.28 & 0.18 \\
\hline 80 & C. buchananii Berger. & & Herb & - & - & - & - & - & - & + & 57.14 & 0.72 \\
\hline 81 & Cortaderia selloana (Schult.) A. \& Gr. & & Herb & - & - & + & + & + & + & - & 42.85 & 0.54 \\
\hline 82 & Calamagrostis acutiflora (Schrad.) DC. & & Herb & - & - & + & + & - & + & - & 14.28 & 0.18 \\
\hline \multicolumn{13}{|c|}{ Dicotyledons } \\
\hline 83 & Habenaria digitata Lindl. & Orchidaceae & Herb & - & - & - & - & - & - & + & 14.28 & 0.18 \\
\hline 84 & Epipactis helleborine (L.) Crantz & & Herb & & & & & & & & 14.28 & 0.18 \\
\hline 85 & Oenothera rosea L Her. ex Aiton & & Herb & - & - & - & - & - & - & + & 14.28 & 0.18 \\
\hline 86 & Zingiber capitatum Roxb. & Zingberceae & Herb & - & - & - & - & - & - & + & 14.28 & 0.18 \\
\hline 87 & Amaranthus viridis $\mathrm{L}$. & Amaranthaceae & Herb & - & - & - & - & - & - & + & 85.71 & 1.08 \\
\hline 88 & A. spinosus $\mathrm{L}$. & & Herb & - & - & - & - & - & - & + & 85.71 & 1.08 \\
\hline 89 & Achyranthes bidentata Blume & & Herb & + & + & + & + & + & + & - & 28.57 & 0.36 \\
\hline 90 & Mangifera indica $\mathrm{L}$. & Anacardiaceae & Herb & + & + & + & + & + & + & - & 42.85 & 0.54 \\
\hline 91 & Hedera nepalensis K.Koch & Araliaceae & Tree & - & - & + & - & - & + & - & 71.42 & 0.90 \\
\hline 92 & Taraxacum officinale Wabb & Asteraceae & Herb & + & + & - & - & - & + & - & 42.85 & 0.54 \\
\hline 93 & Artemisia vulgaris $\mathrm{L}$. & & Herb & + & + & + & + & - & + & - & 57.14 & 0.72 \\
\hline 94 & Bidens bipinnata $\mathrm{L}$. & & Herb & - & - & + & + & + & + & - & 57.14 & 0.72 \\
\hline 95 & Conyza canadensis (L.) Cronquist & & Herb & + & + & + & + & - & - & - & 71.42 & 0.90 \\
\hline 96 & Lactuca sativa $\mathrm{L}$. & & Herb & + & + & + & - & - & + & - & 28.57 & 0.36 \\
\hline 97 & Helianthus annuus L. & & Herb & + & + & + & - & + & + & - & 28.57 & 0.36 \\
\hline 98 & Solidago virgaurea L. & & Herb & - & - & - & - & + & + & - & 57.14 & 0.72 \\
\hline 99 & Sonchus asper (L.) Hill & & Herb & - & - & - & - & + & - & + & 57.14 & 0.72 \\
\hline 100 & Anaphalis spp. & & Herb & + & + & + & - & + & - & - & 14.28 & 0.18 \\
\hline 101 & Lactuca dissecta D.Don & & Herb & - & - & + & + & + & + & - & 28.57 & 0.36 \\
\hline 102 & Achillea millefolium $\mathrm{L}$. & & Herb & - & - & - & - & - & - & + & 42.85 & 0.54 \\
\hline
\end{tabular}




\begin{tabular}{|c|c|c|c|c|c|c|c|c|c|c|c|c|}
\hline 103 & Berberis lycium Royle & Berberidaceae & Herb & - & - & - & + & - & - & + & 57.14 & 0.72 \\
\hline 104 & Trichodesma indicum (L.) Lehm. & Boraginaceae & Shrub & + & + & + & - & - & - & - & 28.57 & 0.36 \\
\hline 105 & Cynoglossum lanceolatum Forssk. & & Herb & - & - & + & + & + & + & - & 57.14 & 0.72 \\
\hline 106 & Lepidium pinnatifidum Ledeb. & Brassicaceae & Herb & + & + & - & - & - & - & - & 57.14 & 0.72 \\
\hline 107 & Buxus papillosa C.K. Schneid. & Buxaceae & Herb & - & - & + & + & + & + & - & 42.85 & 0.54 \\
\hline 108 & Sarcococa pruniformis Lindl. & & Shrub & - & - & + & + & + & + & - & 57.14 & 0.72 \\
\hline 109 & Impatiens spp. & Balsaminaceae & Shrub & - & - & - & + & + & + & - & 14.28 & 0.18 \\
\hline 110 & Commelina benghalensis $\mathrm{L}$. & Commelinaceae & Herb & + & + & + & - & - & + & - & 28.57 & 0.36 \\
\hline 111 & Cuscuta reflexa Roxb. & Cuscutaceae & Herb & - & - & - & - & - & - & + & 14.28 & 0.18 \\
\hline 112 & Solena amplexicaulis (Lam.) Gandhi & Cucurbitaceae & Herb & + & + & - & - & - & - & - & 42.85 & 0.54 \\
\hline 113 & Cucumis sativus $\mathrm{L}$. & & Herb & - & - & - & - & - & - & + & 85.71 & 1.08 \\
\hline 114 & Lagenaria sicernaria (Molina) Standl. & & Herb & + & + & - & - & + & - & - & 28.57 & 0.36 \\
\hline 115 & Momordica charantia $\mathrm{L}$. & & Herb & + & + & + & + & + & + & - & 28.57 & 0.36 \\
\hline 116 & Convolvulus arvensis $\mathrm{L}$. & Convolvulaceae & Herb & - & + & + & - & - & - & - & 28.57 & 0.36 \\
\hline 117 & $\begin{array}{ll}\text { Ipomoea purpurea } & \text { (L.) Roth }\end{array}$ & & Herb & + & + & - & - & - & - & - & 28.57 & 0.36 \\
\hline 118 & Campanula spp & Campanulaceae & Herb & + & + & - & - & - & - & - & 14.28 & 0.18 \\
\hline 119 & Siene spp. & Caryophyllaceae & Herb & - & - & + & + & - & - & - & 14.28 & 0.18 \\
\hline 120 & Chenopodium album $\mathrm{L}$. & Chenopodiaceae & Herb & - & - & - & - & - & - & + & 42.85 & 0.54 \\
\hline 121 & C. murale $\mathrm{L}$. & & Herb & - & - & - & - & - & - & + & 57.14 & 0.72 \\
\hline 122 & Cannabis sativa $\mathrm{L}$. & Cannabinaceae & Herb & + & + & + & - & - & - & - & 28.57 & 0.36 \\
\hline 123 & Vibernum nervosum D.Don & Caprifoliaceae & Herb & + & + & - & - & + & + & - & 14.28 & 0.18 \\
\hline 124 & V. grandiflorum Wall. ex DC. & & Shrub & + & + & - & - & - & - & - & 42.85 & 0.54 \\
\hline 125 & Prosopis sineraria (L.) Druce & Cesaelpinlaceae & Shrub & - & - & - & - & - & - & + & 14.28 & 0.18 \\
\hline 126 & Euphorbia helioscopia L. & Euphorbiaceae & Shrub & + & + & + & - & - & - & - & 42.28 & 0.53 \\
\hline 127 & Diospyros kaki Thunb. & Ebenaceae & Herb & - & - & - & - & - & - & + & 28.57 & 0.36 \\
\hline 128 & D. lotus $\mathrm{L}$. & & Tree & + & + & + & - & - & - & - & 42.85 & 0.54 \\
\hline 129 & Elaeagnus umbellata Thunb. & Elaeagnaceae & Tree & + & - & + & - & - & - & - & 14.28 & 0.18 \\
\hline
\end{tabular}




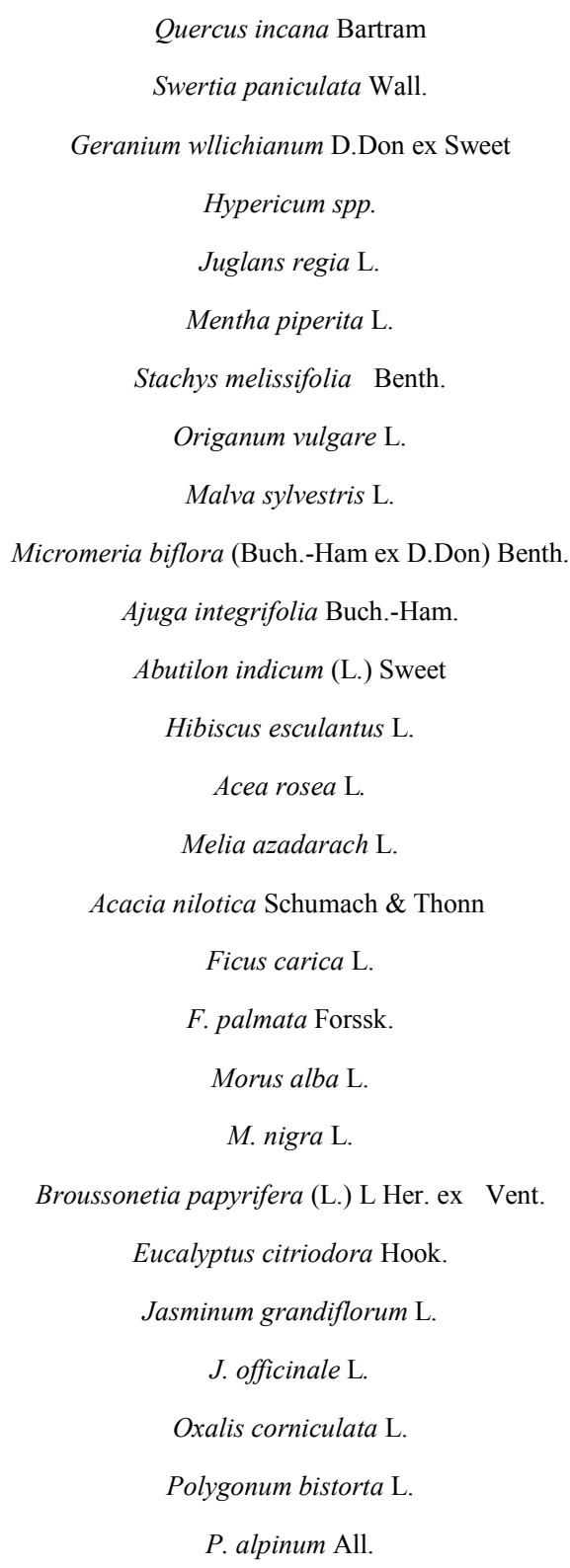

\begin{tabular}{|c|c|c|c|c|c|c|c|c|c|c|}
\hline Fagaceae & Shrub & + & + & + & - & - & - & - & 14.28 & 0.18 \\
\hline Gentianaceae & Tree & - & - & - & - & - & - & + & 14.28 & 0.18 \\
\hline Geraniaceae & Herb & - & - & - & - & - & - & + & 14.28 & 0.18 \\
\hline Hypericaceae & Herb & - & - & - & - & - & - & + & 14.28 & 0.18 \\
\hline Juglandaceae & Herb & - & - & - & - & - & - & + & 42.85 & 0.54 \\
\hline \multirow[t]{3}{*}{ Labiateae } & Tree & - & - & - & - & - & - & + & 28.57 & 0.36 \\
\hline & Herb & + & + & + & - & - & - & - & 42.85 & 0.54 \\
\hline & Herb & + & + & - & - & - & - & - & 28.57 & 0.36 \\
\hline \multirow[t]{6}{*}{ Malvaceae } & Herb & + & + & + & - & - & - & - & 42.85 & 0.54 \\
\hline & Herb & - & + & + & - & - & - & - & 42.85 & 0.54 \\
\hline & Herb & + & + & + & - & - & - & - & 14.28 & 0.18 \\
\hline & Herb & + & + & + & - & - & - & - & 28.57 & 0.36 \\
\hline & Herb & - & - & - & - & - & - & + & 42.85 & 0.54 \\
\hline & Herb & - & - & + & - & - & + & - & 42.85 & 0.54 \\
\hline Meliaceae & Herb & - & - & - & + & + & + & - & 28.57 & 0.36 \\
\hline \multirow[t]{6}{*}{ Moraceae } & Tree & - & - & - & + & + & + & - & 28.57 & 0.36 \\
\hline & Tree & + & + & - & - & - & - & - & 14.28 & 0.18 \\
\hline & Tree & & & & & & & & 42.85 & 0.54 \\
\hline & Tree & - & - & - & - & - & - & - & 28.57 & 0.36 \\
\hline & Tree & + & + & + & - & - & - & + & 57.14 & 0.72 \\
\hline & Tree & - & - & + & + & - & + & - & 14.28 & 0.18 \\
\hline Myrtaceae & Tree & + & + & + & - & - & + & - & 42.85 & 0.54 \\
\hline \multirow[t]{2}{*}{ Oleaceae } & Tree & - & - & - & - & - & - & + & 57.14 & 0.72 \\
\hline & Shrub & - & - & + & + & - & + & - & 57.14 & 0.72 \\
\hline Oxalidaceae & Shrub & - & + & + & - & + & + & - & 14.28 & 0.18 \\
\hline \multirow[t]{2}{*}{ Polygonaceae } & Herb & - & - & + & + & + & + & - & 57.14 & 0.72 \\
\hline & Herb & - & - & - & - & - & - & + & 14.28 & 0.18 \\
\hline
\end{tabular}




\begin{tabular}{|c|c|}
\hline 157 & Rumex dentatus $\mathrm{L}$. \\
\hline 158 & Rumex nepalensis Spreng \\
\hline 159 & Persicaria nepalensis (Meisn.) Miyabe \\
\hline 160 & Androsace rotundifolia Hardw. \\
\hline 161 & Medicago lupulina $\mathrm{L}$. \\
\hline 162 & Phaseolus lunatus L. \\
\hline 163 & Robinia psudoacacia $\mathrm{L}$. \\
\hline 164 & Indigofera heterantha Brandis \\
\hline 165 & Lathyrus aphaca L. \\
\hline 166 & Trigonella foenum-graceum $\mathrm{L}$. \\
\hline 167 & Crotalaria medcaginea Lam. \\
\hline 168 & Punica granatum $\mathrm{L}$. \\
\hline 169 & Plantago lanceolata $\mathrm{L}$. \\
\hline 170 & Geum elatum Wall. ex G.Don \\
\hline 171 & Eriobotrya japonica (Thunb.) Lindl. \\
\hline 172 & Fragaria nubicola (Hook.f.) Lindl. Ex Lacaita \\
\hline 173 & Rosa indica \\
\hline 174 & Potentilla nepalensis Hook.f. \\
\hline 175 & P. fruticosa $\mathrm{L}$. \\
\hline 176 & Duchesnea indica (Andrews) Focke \\
\hline 177 & Malus pumila Mill. \\
\hline 178 & Prunus persica (L.) Stokes \\
\hline 179 & P. armeniaca $\mathrm{L}$. \\
\hline 180 & Pyrus pashia Buch. -Ham. ex D.Don \\
\hline 181 & Prunus domestica L. \\
\hline 182 & Prunus bokhariensis Royle \\
\hline 183 & Malus sylvestris Mill. \\
\hline
\end{tabular}

\begin{tabular}{|c|c|c|c|c|c|c|c|c|c|c|}
\hline & Herb & + & + & + & - & - & + & - & 57.14 & 0.72 \\
\hline & Herb & - & - & - & - & - & - & + & 14.28 & 0.18 \\
\hline & Herb & + & + & + & + & - & + & - & 57.14 & 0.72 \\
\hline Primulaceae & Herb & + & + & - & - & - & - & + & 14.28 & 0.18 \\
\hline Paplionaceae & Herb & + & + & + & + & - & + & - & 28.57 & 0.36 \\
\hline & Herb & - & - & - & - & - & - & + & 57.14 & 0.72 \\
\hline & Herb & - & - & - & + & + & - & - & 57.14 & 0.72 \\
\hline & Tree & + & + & + & - & - & + & - & 14.28 & 0.18 \\
\hline & Shrub & - & - & + & + & + & + & - & 42.85 & 0.54 \\
\hline & Herb & - & - & - & - & - & - & + & 28.57 & 0.36 \\
\hline & Herb & + & + & - & - & - & + & - & 28.57 & 0.36 \\
\hline Punicaceae & Shrub & - & - & - & + & - & + & - & 42.85 & 0.54 \\
\hline Plantaginaceae & Tree & - & + & - & - & - & + & - & 14.28 & 0.18 \\
\hline Rosaceae & Herb & + & + & - & - & + & - & - & 14.28 & 0.18 \\
\hline & Herb & - & - & - & - & - & - & + & 28.57 & 0.36 \\
\hline & Tree & - & - & - & - & - & - & + & 14.28 & 0.18 \\
\hline & Herb & - & + & - & - & + & - & - & 42.85 & 0.54 \\
\hline & Shrub & - & - & - & - & - & - & + & 42.85 & 0.54 \\
\hline & Herb & - & - & - & + & + & + & - & 57.14 & 0.72 \\
\hline & Herb & - & - & + & + & - & + & - & 14.28 & 0.18 \\
\hline & Herb & - & + & + & + & + & + & - & 57.14 & 0.72 \\
\hline & Tree & - & - & - & - & - & - & + & 57.14 & 0.72 \\
\hline & Tree & - & - & + & + & + & + & - & 57.14 & 0.72 \\
\hline & Tree & + & + & + & - & - & + & - & 57.14 & 0.72 \\
\hline & Tree & + & + & + & - & - & + & - & 42.85 & 0.54 \\
\hline & Tree & + & - & + & + & - & + & - & 57.14 & 0.72 \\
\hline & Tree & - & - & - & + & + & + & - & 28.57 & 0.36 \\
\hline
\end{tabular}




\begin{tabular}{|c|c|c|c|c|c|c|c|c|c|c|c|c|}
\hline 184 & Zanthoxylem allatum Roxb. & Rutaceae & Tree & - & - & + & + & + & + & - & 42.85 & 0.54 \\
\hline 185 & Galium aparine $\mathrm{L}$. & Rubiaceae & Shrub & + & - & - & + & - & - & - & 57.14 & 0.72 \\
\hline 186 & G. Boreale L. & & Herb & - & + & + & - & + & - & - & 42.85 & 0.54 \\
\hline 187 & Glematic spp. & Ranunculaceae & Herb & - & - & + & + & + & + & - & 14.28 & 0.18 \\
\hline 188 & Ranunculus muricatus L. & & Shrub & - & + & - & - & + & + & - & 42.85 & 0.54 \\
\hline 189 & Thalictrum foliolosum DC. & & Herb & - & - & - & - & - & - & + & 57.14 & 0.72 \\
\hline 190 & Solanum nigrum L. & Solanaceae & Herb & + & + & + & - & - & - & - & 42.85 & 0.54 \\
\hline 191 & S. tuberosum L. & & Herb & - & + & + & - & + & + & - & 42.85 & 0.54 \\
\hline 192 & Lycopersicum esculentum Mill. & & Herb & - & - & - & + & + & + & - & 42.85 & 0.54 \\
\hline 193 & Cestrum nocturnum $\mathrm{L}$. & & Herb & - & - & + & - & + & + & - & 57.14 & 0.72 \\
\hline 194 & Verbascum thapsus $\mathrm{L}$. & Scrophyulariaceae & Shrub & + & + & - & - & - & + & - & 57.14 & 0.72 \\
\hline 195 & Populus nigra $\mathrm{L}$. & Salicaceae & Herb & + & + & - & - & + & + & - & 42.85 & 0.54 \\
\hline 196 & P. alba $\mathrm{L}$. & & Tree & + & + & + & + & - & - & - & 28.57 & 0.36 \\
\hline 197 & Anethum graveolens L. & Simaroubaceae & Tree & - & + & + & - & + & - & - & 57.14 & 0.72 \\
\hline 198 & Urtica dioica $\mathrm{L}$. & Urticaceae & Herb & - & - & - & - & - & - & + & 42.85 & 0.54 \\
\hline 199 & Viola odorata L. & Violaceae & Shrub & - & + & - & - & - & + & - & 42.85 & 0.54 \\
\hline 200 & Vitis vinifera $\mathrm{L}$. & Vitaceae & Herb & + & + & - & - & - & - & - & 42.85 & 0.54 \\
\hline
\end{tabular}




\section{Results and Discussion}

\subsection{Floristic Composition}

During the study, the whole flora was explored from subtropical foothills of Kahutta to alpine pasture of Kiran. A total of 200 plant species of 170 genera and 65 families were reported from study area. The study area was divided into seven subunits (Figure 1), based on altitudinal variations. Richest site was Mahmood Galli with 101 species (dicots 55, monocots 35 , pteridophytes 10 and gymnosperm 1 ) followed by Toliper 100 spp. (dicots 55, monocots 31, pteridophytes 13 and gymnosperms 1), Lasdana, 85 spp. (dicots 47 , monocots 35, pteridophytes 6 and gymnosperms 2), Plungi 81spp. (dicots 51, monocots13, pteridophytes 16 and gymnosperm 1), Khurshidabad, 74 spp. (dicots 34, monocots 21 , pteridophytes 19), Kahutta, 70 spp. (dicots 36 , monocots 15, pteridophytes 19 ) and Kiran 46 Spp. dicots 34, gymnosperm 3).

The checklist comprised of 167 angiosperms, (dicots 118 and monocots 49), 29 pteridophytes and 4 gymnosperms (Figure 3). Dominating life forms in all seven sites were herbs (156 spp.), followed by shrubs (29 spp.) and trees (15 spp., Figure 2). The leading family was Poaceae with 42 species (Figure 4) followed by Rosaceae and Asteraceae (14, $11 \mathrm{spp}$.), other families were with fewer number of species.

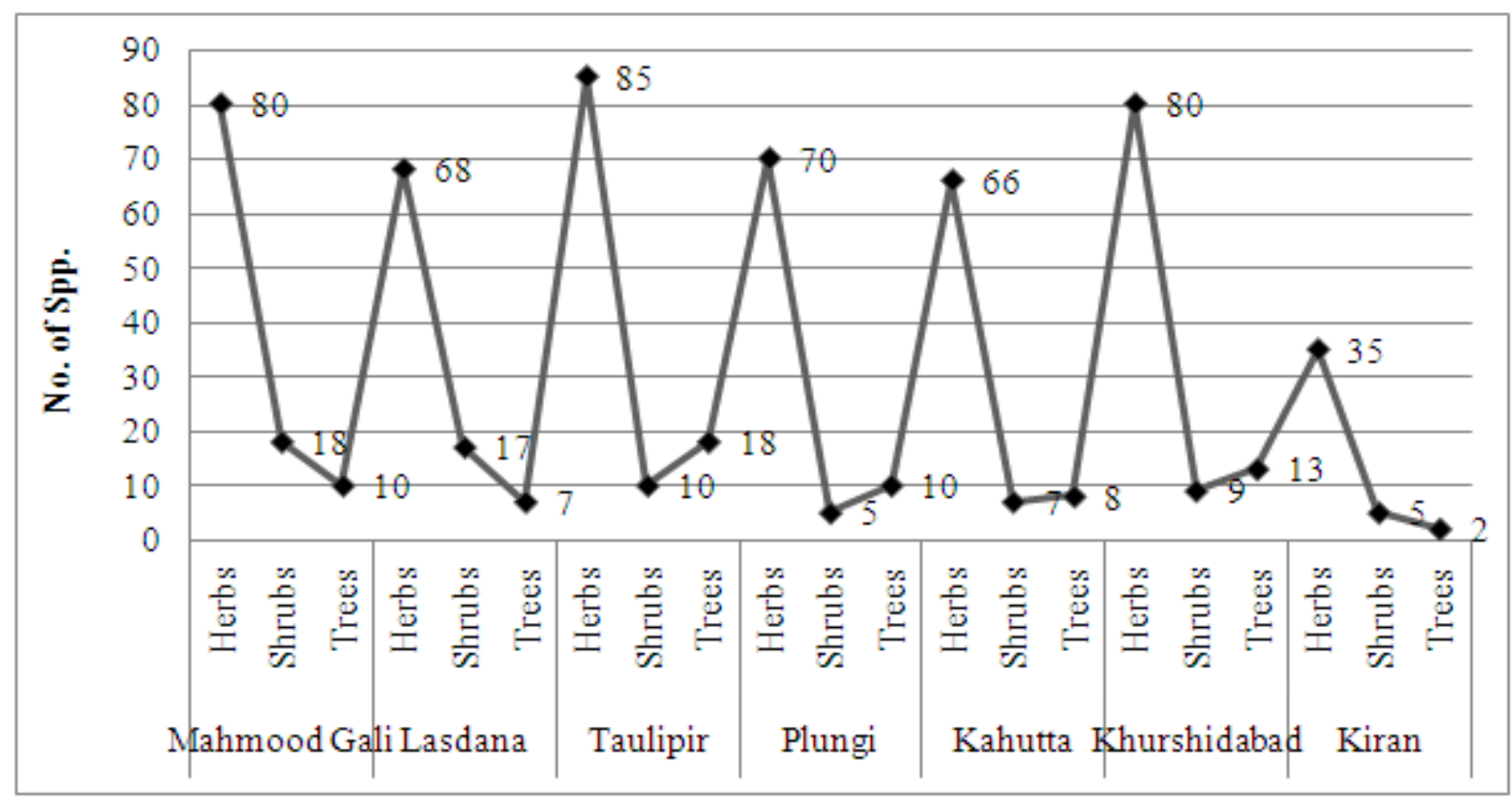

Figure 2. Life forms of various localities in the District Bagh, AJ\&K.

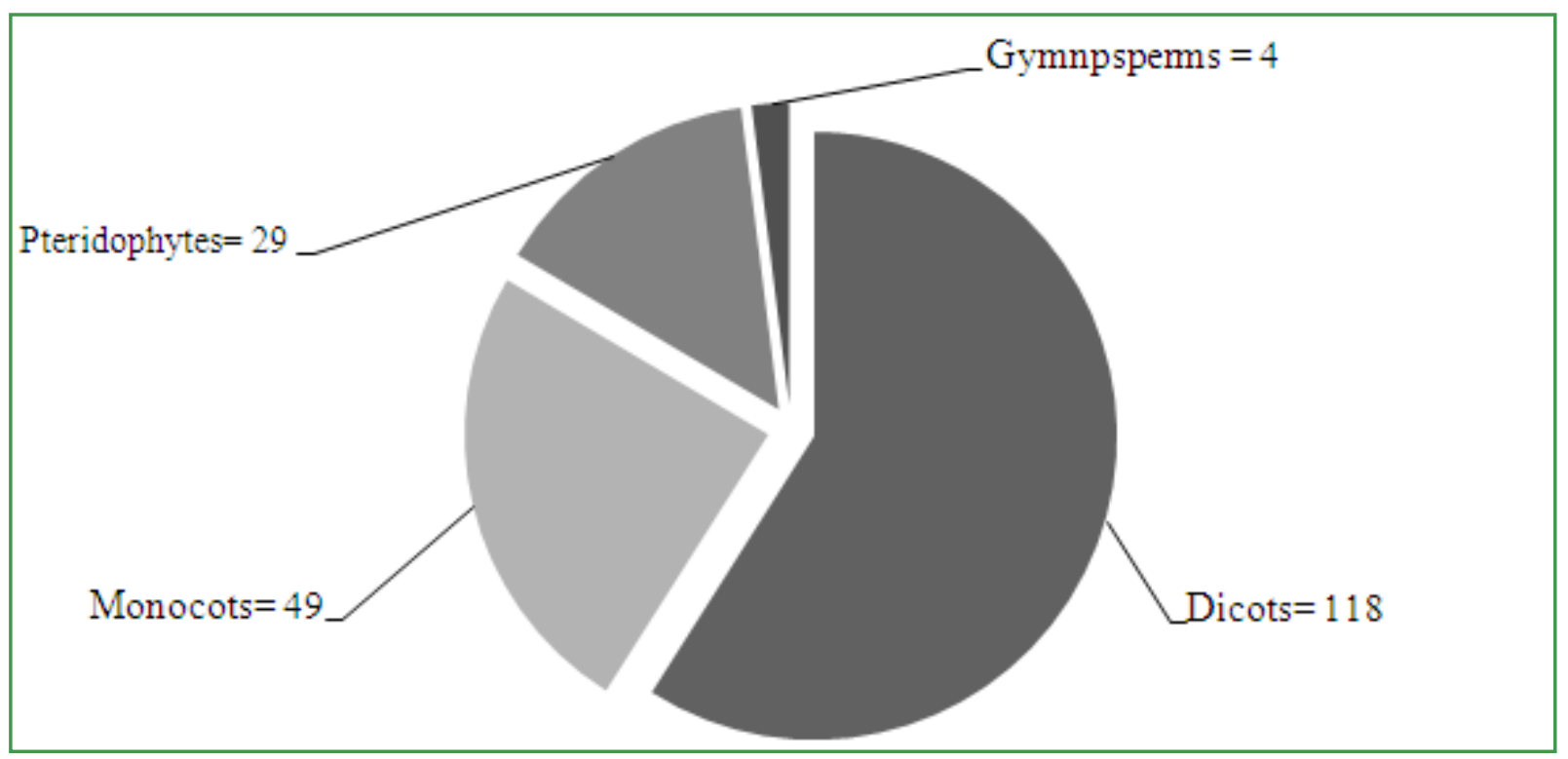

Figure 3. Plant groups of the District Bagh, AJ\&K. 


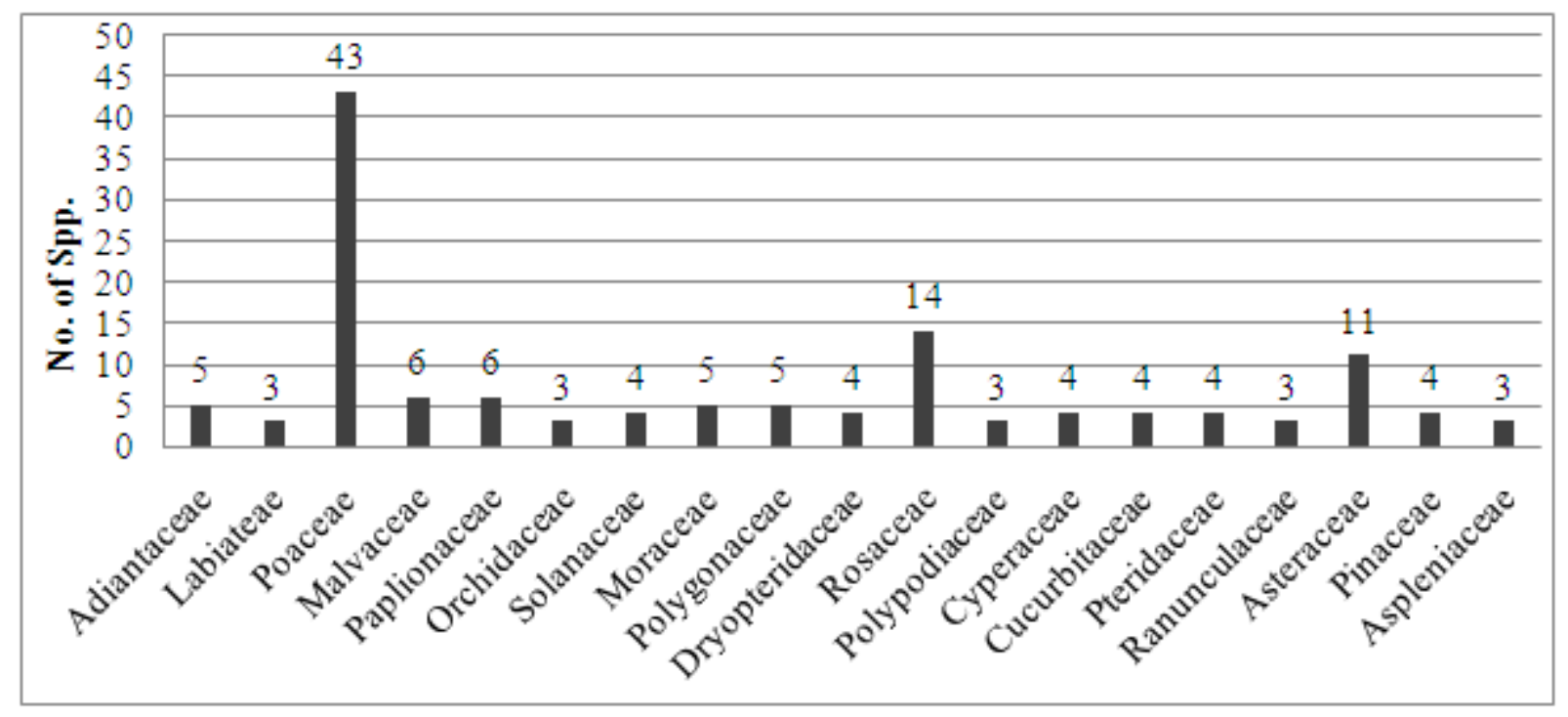

Figure 4. Dominating families of the District Bagh, AJ\&K.

\subsection{Percentage Distribution}

Among pteridophytes, Polystichum squarrosum had highest distribution percentage $85.71 \%$ and high relative frequency $1.08 \%$ (Table 1), while Lepisorus clathratus and Pyrrosia mollis have least percentage distribution $4.28 \%$ with low relative frequency $0.18 \%$. All the reported species of gymnosperm were trees. Pinus wallichiana had highest distribution percentage $57.14 \%$ and relative frequency $0.72 \%$ while Cedrus deodara and Pinus roxburgii have least distribution percentage $14.28 \%$ and low relative frequency $0.18 \%$. Aristida abnormis had highest distribution percentage with $71.42 \%$ and relative frequency $0.92 \%$ while Zizania aquatica and Themeda anathera and Briza media have least distribution percentage with $14.28 \%$ and low relative frequency $0.18 \%$. In dicots, Amaranthus viridis, Amaranthus spinosus and Cucumis sativus had highest distribution percentage with $85.71 \%$ and high relative frequency $1.08 \%$ while Oxalis corniculata, Quercus incana and Fragaria nubicola have least frequency with $14.28 \%$.

\subsection{Altitudinal Variation}

It was observed that percentage distribution (frequency) decreases with the increase in altitude while it was high in the low altitude. Similar findings were reported by [11]. The experiential decrease in species distribution is due to deforestation, human interaction, collections of medicinal plants and quick disappearance of annual plants because of cold conditions [12]. Species diversity was high in the tree layer in the middle part of the altitudinal gradient. It decreases both towards upper and lower altitude, which was due to deforestation, human interaction, encroachment pressure, low number of species and soil erosion.

\subsection{Threatened Flora of Kashmir}

Medicinal flora of Senhsa, District Kotli (adjacent area of
District Bagh) is under serious threats [1]. Ajuga bracteosa, Mallottus philippinensis, Butea monosperma, and Zanthoxylum armatum are critically endangered not only locally, but also in the whole region. Among endangered species, Cissus carnosa, Juglans regia, Olea ferruginaea,, Phyllanthus emblica, Viola canescens are the notable species, which are at high risk of being endangered [13]. An effective conservation plan cannot be implemented without knowing the indigenous flora, habitat ecology and anthropogenic factors, affecting the population of plant species, particularly those of vulnerable and threatened either locally or internationally [14]. In Neelum valley, Saussurea lappa, Aconitum heterophyllum, Geranium wallichianum, Jurinea dolomiaea, Ajuga bracteosa, Bistorta amplexicaule, and Berberis lyceum are on the verge of extinction due to high rate of utilization [15]. There has been a rapidly increasing interest in the effects of species richness on community productivity in recent years. The relationship between species diversity and ecosystem function, combined with the worldwide loss of species, has become one issue that has attracted substantial attention [16]. Decreasing primary productivity and changes in the structure of plant communities have been caused by the destruction of biodiversity, unreasonable exploitation and overgrazing of grassland resources in some areas, with consequent impacts on human society. Productivity is one of the important modalities by which to weigh up ecosystem functions [17], so a healthier knowledge of the association between plant-species diversity and ecosystem functioning would help to indulgent whole ecosystems.

\section{Conclusion}

The interest behind the selection of the area was its thick vegetation and great altitudinal variation, ranging from subtropical to alpine. The area lies in temperate zone, 
characterized by cold climate. Plant resources are limited that require efficient, wise and sustainable management and conservation strategies. Necessary steps should be taken not only to store the original vegetation but also to improve it. Overgrazing and deforestation should be abridged. Forest management practices that benefit biodiversity conservation should be encouraged. Awareness programs at grass root level should be introduced.

\section{REFERENCES}

[1] K.S Ahmad, W. K. Kiyani, M. Hameed, F. Ahmad, T. Nawaz. Floristic diversity and ethnobotany of Senhsa, District Kotli, Azad Jammu \& Kashmir (Pakistan). Pak. J. Bot, 44(SI): 195-201, 2012a.

[2] Schuster, B. Diekmann, M. 2005. Species richness and environmental correlates in deciduous forests Northwest Germany. For. Ecol. Manag, 206: 197-205.

[3] Titshall, L.W., Connor, O.T.G. Morris, C.D. 2000. Effect of long-term exclusion of fire and herbivory on the soils and vegetation of sour grassland. Afri. J. Range Forage Sci, 17: $70-80$.

[4] Hussain, M., Ali, A. 2006. Spatial and seasonal variation in the species composition of herbaceous vegetation indigenous to Soon valley in the Salt Range of Pakistan. Pak. J. Agric. Sci, 43: 43-49.

[5] Peer, T., Guber, G.P., Millingard J.A., Hussain, F. 2007. Phytosociology, structure and diversity of the steppes vegetation in the mountains of Northern Pakistan. Phytocoenologia, 37: 1-65.

[6] Ali, S.I. The significance of flora with special reference to Pakistan. Pak. J. Bot, 40(30): 967-971, 2008.

[7] M. Hameed, T. Nawaz, M. Ashraf, F. Ahmad, K. S. Ahmad, M. S. A. Ahmad, S. H., Raza, M. Hussain, I. Ahmad. Floral biodiversity and conservation status of the Himalayan foothill region, Punjab. Pak. J. Bot, 44: 143-149, 2012.

[8] Anonymous. www.pm.ajk.gov. Official website of AJ\& K Government. 2008.

[9] Nasir, E. Ali, S.I. 1970-1989. Flora of Pakistan. National Herbarium, PARC, Islamabad.

[10] Ali, S.I., M. Qaisar, Flora of Pakistan. Department of Botany, University of Karachi and National Herbarium, PARC, Islamabad, 1992-2007.

[11] Malik, Z.H. Comparative study of the Vegetation of the Ganga Choti and Badori Hills, Distt. Bagh, Azad Jammu and Kashmir, with special reference to range conditions, $\mathrm{Ph}$. D thesis, Dept. Bot. University of Peshawar, 2005.

[12] Ram, J., Kumar, A., Bhatt, J. 2004. Plant diversity in six forest types of Uttaranchal, Central Himalaya, India. Curr. Sci, 86(7): 208-215.

[13] Nawaz, T., Hameed, M., Ashraf, M., Ahmad, F., Ahmad, M.S.A., Hussain M., Ahmad, I., Younis, A., K.S. Ahmad. Diversity and conservation status of economically important flora of the Salt Range, Pakistan. Pak. J. Bot, 44: 203-211, 2012.

[14] Qureshi, R., G.R. Bhatti and R.A. Memon. Ethnomedicinal uses of herbs from Nara Desert, Pakistan. Pak. J. Bot., 42(2): $839-851,2010$.

[15] K. S. Ahmad, R. Qureshi, M. Hameed, F. Ahmad, T. Nawaz. Conservation assessment and medicinal importance of some plants resources from Sharda, Neelum valley, Azad Jammu and Kashmir. Int. J. Agric. Biol, 14: 997-1000, 2012 b.

[16] Zhang, D.Y. 2000. 'Researches on theoretical ecology.' (Higher Education Press: Beijing).

[17] Tilman, D. The ecological consequences of changes in biodiversity: a search for general principles. Ecology, 80: 1455-1474, 1999. 